\title{
A Time-Series Model of Phase Amplitude Cross Frequency Coupling and Comparison of Spectral Characteristics with Neural Data
}

\author{
Kyle Q. Lepage and Sujith Vijayan \\ The Department of Mathematics \& Statistics, Boston University, 111 Cummington Mall, Boston, MA 02215, USA
}

Correspondence should be addressed to Kyle Q. Lepage; lepage@math.bu.edu

Received 25 January 2015; Accepted 5 March 2015

Academic Editor: Tjeerd Boonstra

Copyright (C) 2015 K. Q. Lepage and S. Vijayan. This is an open access article distributed under the Creative Commons Attribution License, which permits unrestricted use, distribution, and reproduction in any medium, provided the original work is properly cited.

\begin{abstract}
Stochastic processes that exhibit cross-frequency coupling (CFC) are introduced. The ability of these processes to model observed CFC in neural recordings is investigated by comparison with published spectra. One of the proposed models, based on multiplying a pulsatile function of a low-frequency oscillation $(\theta)$ with an unobserved and high-frequency component, yields a process with a spectrum that is consistent with observation. Other models, such as those employing a biphasic pulsatile function of a low-frequency oscillation, are demonstrated to be less suitable. We introduce the full stochastic process time series model as a summation of three component weak-sense stationary (WSS) processes, namely, $\theta, \gamma$, and $\eta$, with $\eta$ a $1 / f^{\alpha}$ noise process. The $\gamma$ process is constructed as a product of a latent and unobserved high-frequency process $x$ with a function of the lagged, low-frequency oscillatory component $(\theta)$. After demonstrating that the model process is WSS, an appropriate method of simulation is introduced based upon the WSS property. This work may be of interest to researchers seeking to connect inhibitory and excitatory dynamics directly to observation in a model that accounts for known temporal dependence or to researchers seeking to examine what can occur in a multiplicative time-domain CFC mechanism.
\end{abstract}

\section{Introduction}

Cross frequency coupling (CFC) is a statistical relation between the phase or amplitude of a low frequency and the phase or amplitude of a high frequency. In this work, focus is placed upon phase-amplitude CFC, which can be thought of as the correlation of the amplitude of a relatively highfrequency oscillation $(\gamma)$ with the phase of a lower frequency oscillation $(\theta)$.

The rationale behind this proposal is based on the experimental observations that (i) relatively slow frequency oscillations tend to be coordinated over large regions of neural tissue, unlike higher frequency oscillations $[1,2]$, and (ii) oscillatory activity reflects changes in the excitability of neural tissue [3]. Hence, a lower frequency oscillation may provide time intervals in which high-frequency activity may occur and may consequently coordinate high-frequency oscillations that are spatially disparate.
Recently, the theoretical interest in phase-amplitude CFC has been reinforced. Recent observations of the phenomenon have occurred in varied species [4-6], brain regions [7-9], and states of vigilance [10]. Experimental studies have shown that the nature of phase-amplitude CFC can be altered by predictive cues and attentional demands; the phase of the low-frequency oscillation can be reset such that a stimulus of attentional interest arrives at the phase of maximal excitability [11]. Furthermore, phase-amplitude CFC has been implicated in learning and memory $[6,12]$, and the dynamics of phaseamplitude CFC have been shown to change over the course of a cognitive task [8].

In this work, in a fashion akin to that classically employed for parametrically modeling the spectra of timeseries, stochastic time-series models are constructed which exhibit cross frequency coupling (see, e.g., [13-15]). Through simulation it is shown that these models can produce timeseries that exhibit CFC similar to that exhibited in neural 
recordings. Some mathematical properties of these models are given, and some consequences are discussed.

\section{Methodology}

Stochastic processes exhibiting cross frequency coupling are introduced. The ability for these processes to model observed cross frequency coupling in neural recordings is investigated and mathematical properties of the new models are given. Investigations are conducted through the use of mathematical analysis and simulation.

2.1. Model Specification. The stochastic, or random, processes introduced in this work model CFC phenomena in the following way. For each recorded measurement a random variable is introduced; the measurement is modeled as a realization of this random variable. The collection of random variables comprises a discrete-time random process. The observed time-series is modeled as the corresponding collection of realizations of each of the random variables in the random, or stochastic, process. This is the standard setup in classical time-series analysis [13] and it includes the independent and identically distributed (IID) random sample as a special case.

Each of the random processes is characterized by all of the possible moments between the random variables; in this work consideration will be restricted to random processes whose joint distributions are Gaussian. In this situation, specification of the first two joint moments completely specifies the model.

Without restriction upon the time dependence of pairwise correlation, the number of unique pair-wise correlations increases quadratically with every new observation (in a single trial). This is a more challenging regime to perform inference than is typically considered, as in this case the number of unknowns is growing rapidly with increasing observation length. Contemporary work deals with this issue by recording many trials, or by using models with other restrictions.

Here, as is often customary, the introduced models are weak-sense stationary (WSS) random processes [13-15]. The weak-sense stationary property implies that the correlation between any pair of random variables in the process does not depend upon absolute time, but, rather, only upon the difference in the times associated with the pair. It also implies that the mean of each of the random variables in the process is equal; thus the process mean is also independent of time. Gaussian weak-sense stationary random processes are amongst the simplest of time-series models and the number of pair-wise correlations for these processes is of the same order as the number of measurements.

In this work, the random processes exhibiting CFC are constructed from component WSS processes modeling $\theta$-rhythm, $\gamma$-rhythm, background activity, and sensor noise. The mean of these processes is taken to be zero, consistent with randomly observed neural phenomena (nonevoked). Based upon observed spectra, the autocorrelation of the $\theta, \gamma$, and noise components is specified in the Fourier domain. Based upon the discrete-time analog of the Wiener-Khintchine theorem, the autocorrelation of each of these component processes is obtained by inverse discretetime Fourier transforming the specified spectra [13].

2.1.1. The Component Processes. Let $t$ be the integer-valued time-index of the length $n$ WSS zero-mean random process $\theta$ with autocovariance sequence $r^{(\theta)}$ :

$$
\begin{aligned}
r_{\tau}^{(\theta)} & =E\left\{\theta_{t} \theta_{t+\tau}\right\}-E\left\{\theta_{t}\right\} E\left\{\theta_{t+\tau}\right\}, \\
& =E\left\{\theta_{t} \theta_{t+\tau}\right\} .
\end{aligned}
$$

Here $E\{X\}$ denotes the expected value of the random variable $X$. Similarly, specify WSS zero-mean processes for the $\gamma$ rhythm and noise, $\eta$, components of the model. That is,

$$
\begin{aligned}
& r_{\tau}^{(\gamma)}=E\left\{\gamma_{t} \gamma_{t+\tau}\right\}, \\
& r_{\tau}^{(\eta)}=E\left\{\eta_{t} \eta_{t+\tau}\right\} .
\end{aligned}
$$

Further, specify the $\theta$ and $\eta$ components as uncorrelated. Because both $\theta$ and $\eta$ are also zero-mean, it follows that $E\left\{\theta_{t} \eta_{t^{\prime}}\right\}$ is equal to zero. These components are jointly Gaussian, uncorrelated, and hence they are independent. The components $\theta$ and $\gamma$ are linked to model CFC. This linking and its consequence are discussed in Section 2.1.2. It remains to specify the autocovariance sequences $r^{(\theta)}, r^{(\gamma)}$, and $r^{(\eta)}$. As described, this is accomplished by specificying their respective spectra, $S^{(\theta)}, S^{(\gamma)}$, and $S^{(\eta)}$, and using the example relation obtained by applying the discrete-time analog to the Wiener-Khintchine theorem [13]:

$$
r_{\tau}^{(\theta)}=\Delta \int_{-f_{N}}^{f_{N}} S_{\theta}(f) e^{i 2 \pi f \tau \Delta} d f .
$$

Here $f_{N}$ is the Nyquist frequency, equal to $(2 \Delta)^{-1}$ (in $\mathrm{Hz}$ ), specified in terms of the sample period $\Delta$ (in s). Figures 1 and 2 depict the specified model autocovariance sequences and spectra for the $\theta$ and $\eta$ components (resp.). The $\gamma$ component is further detailed in Section 2.1.2.

\subsubsection{Specification of $\gamma$ Exhibiting Cross Frequency Coupling.} In this work, CFC is specified by equating $\gamma$ to a function $f$, relating $\theta$ to an unobserved, latent random process $x$. Let $x$ be a zero-mean, WSS, random process possessing a spectrum $S_{x}$, such that $S_{x}$ is near zero outside of the frequency interval associated with a $\gamma$-rhythm. Candidate frequency intervals include low- $\gamma(30 \mathrm{~Hz}-50 \mathrm{~Hz})$ and high- $\gamma(100 \mathrm{~Hz}-140 \mathrm{~Hz})$. (These ranges can differ depending on the lab, species, and neural area. In [4], the low- $\gamma$ frequency interval is specified to be $30 \mathrm{~Hz}-50 \mathrm{~Hz}$, and the high- $\gamma$ frequency interval is specified to be $80 \mathrm{~Hz}-150 \mathrm{~Hz}$.) Consider

$$
\gamma_{t}=f\left(\boldsymbol{\theta}_{t}, x_{t}\right)
$$

where $\boldsymbol{\theta}_{t}$ is a vector of random variables belonging to the $\theta$ process. The $j$ th element $\left(\boldsymbol{\theta}_{t}\right)_{j}$ of the random vector $\boldsymbol{\theta}_{t}$ is equal to $\theta_{t+j}$. The function $f$ can be chosen to model various types of CFC. Together the process $x$ and the function $f$ control 


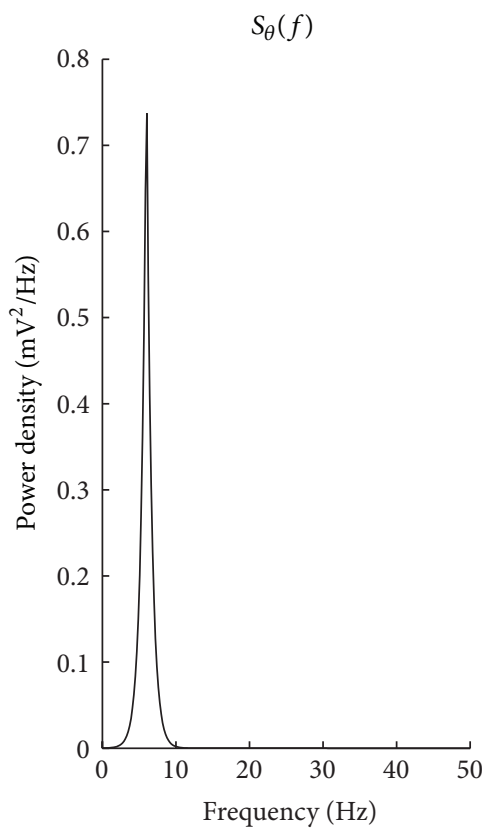

(a)

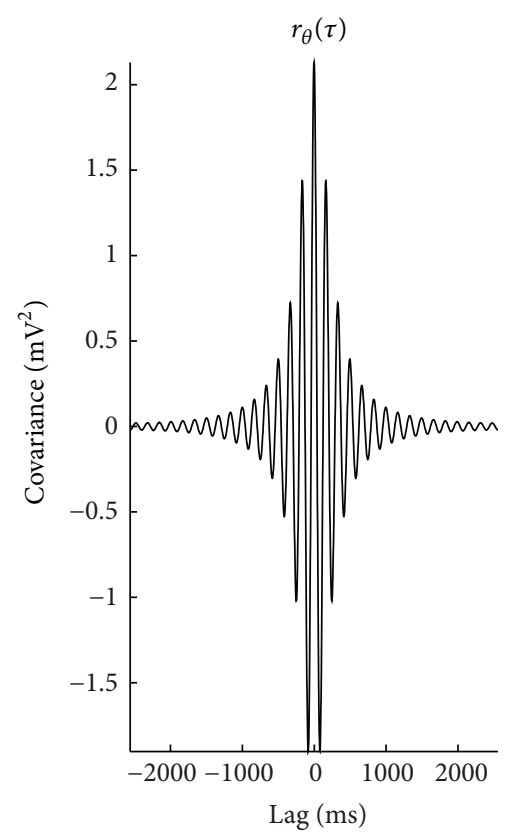

(b)

FIGURE 1: (a) $S_{\theta}$ specified as a $2 \mathrm{~Hz}$ peak exhibited in many neural recordings centered at $6 \mathrm{~Hz}$. (b) The corresponding autocovariance sequence $r^{(\theta)}$.

the dependence of $\gamma$ upon $\theta$. The relation (4) is general and can be used to model many types of CFC. For example, when

$$
\gamma_{t}=\theta_{t+\tau} x_{t}
$$

$\gamma$ exhibits a phase shifted and sinusoidal variance (see Figure 3). When

$$
\gamma_{t}=x_{t} \sum_{k=0}^{K} w_{k} \theta_{t+\tau}^{k} \text {, }
$$

$\gamma$ exhibits a punctate interval of increased variance centered upon the prefered phase $\phi_{p}, \phi_{p}=\tau /\left(2 \pi f_{\theta}\right)$ (for the $K$ equal to 4 case, with $w_{0}=0.4783, w_{1}=0.2625, w_{2}=0.0933, w_{3}=$ 0.0292 , and $w_{4}=0.0058$, see Figure 3 ). (To be pulsatile the Fourier transform of $\gamma$ must have peaks spaced by $f_{0}$. Here this is accomplished by summing $\theta$ taken to higher powers). When $\theta_{t}$ is squared in (6),

$$
\gamma_{t}=x_{t} \sum_{k=0}^{K} w_{k} \theta_{t+\tau}^{2 k}
$$

and biphasic coupling is modeled (see Figure $3, K=4$, and $w_{k}$ as specified in Figure 3). Note that doubling the power of $\theta$ in (7) doubles the harmonic frequency spacing present in the $\gamma$ specified in (6). In the Appendix it is shown that the inverse tranform of a sequence with four harmonics in the frequency domain has a period equal to the inverse of the harmonic spacing.
2.1.3. The Complete Time-Series Model. The model random process $y$ generating the observed time-series is specified as the sum of the $\theta, \gamma$, and $\eta$ components. Consider

$$
y_{t}=\theta_{t}+\gamma_{t}+\eta_{t}
$$

2.2. Properties of the Model. Mathematical properties resulting from the specification (8) are explored by calculation.

2.2.1. The Computation of $E\left\{\gamma_{t}\right\}$. By definition the mean, $\mu_{t}^{(\gamma)}$, of the $\gamma$ component is

$$
\begin{aligned}
\mu_{t}^{(\gamma)} & =E\left\{\gamma_{t}\right\}, \\
& =E\left\{\theta_{t+\tau} x_{t}\right\}, \\
& \left.=E\left\{\theta_{t_{\tau}}\right\} E\left\{x_{t}\right\}, \quad \text { (independence of } \theta \text { and } x\right) \\
& =0 .
\end{aligned}
$$

2.2.2. The Computation of $E\left\{y_{t}\right\}$. By definition, the process mean, $\mu^{(y)}$, evaluated at time-index $t$ is

$$
\begin{aligned}
\mu_{t}^{(y)} & =E\left\{y_{t}\right\}, \\
& =E\left\{\theta_{t}+\gamma_{t}+\eta_{t}\right\}, \\
& =0 .
\end{aligned}
$$

Since all three components $\theta, \gamma$, and $\eta$ are mean-zero processes. Thus, the model process $y$ is also mean-zero. 


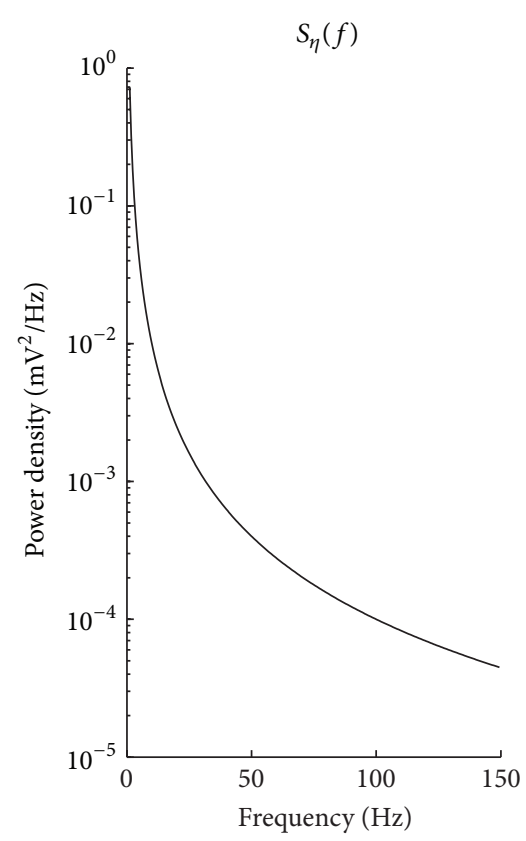

(a)

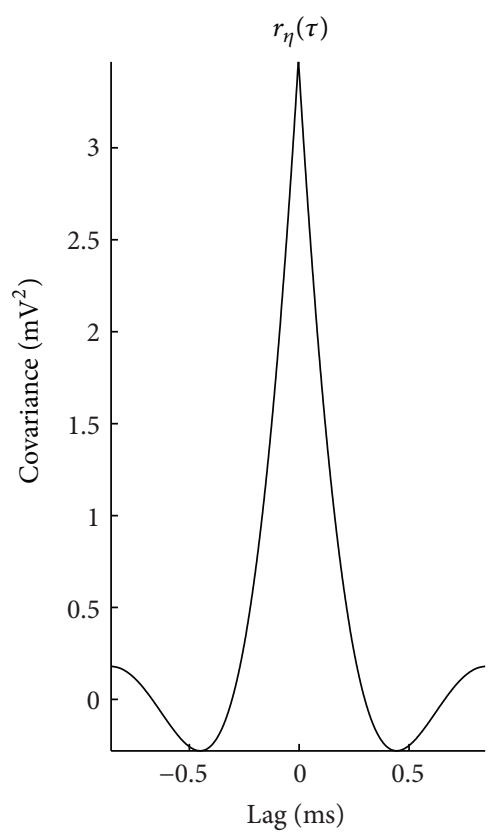

(b)

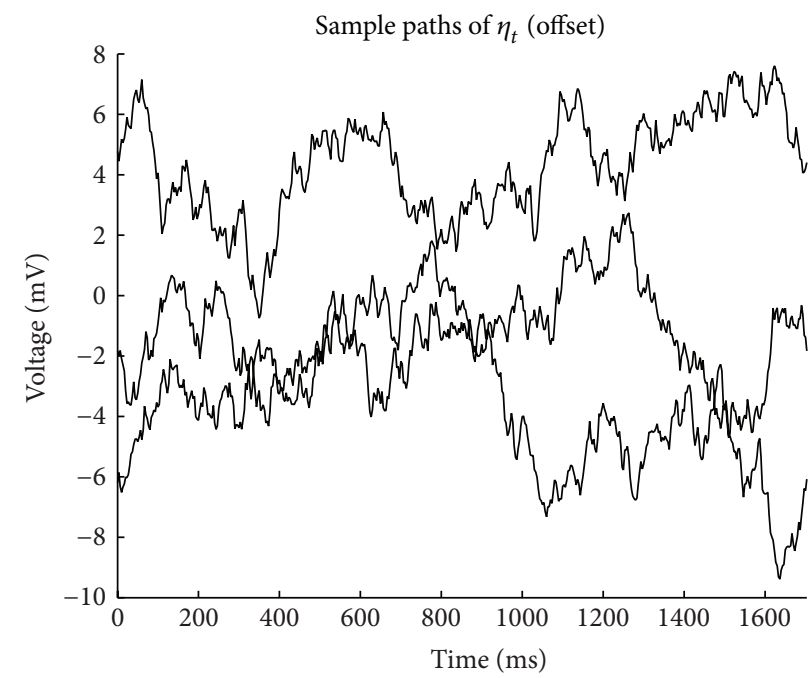

(c)

FIGURE 2: (a) $S_{\eta}$ specified to be pink noise exhibiting the $1 / f^{\alpha}$ decay with increasing frequency typically observed in neural recordings. (b) The corresponding autocovariance sequence $r^{(\eta)}$. Here $\alpha$ is equal to 2. (c) Sample paths of $\eta$.

2.2.3. The Computation of $r_{\tau}^{(\gamma)}$. By construction $\theta, x$, and $\eta$ are WSS random processes. To establish $\gamma$ as a weaksense stationary process, it is necessary to compute the autocovariance function $r^{(\gamma)}$. Begin with the definition for the general autocovariance function evaluated at the time indices $t$ and $t+\tau$. One has

$$
\begin{aligned}
r_{t, t+\tau^{\prime}}^{(\gamma)} & =E\left\{\gamma_{t} \gamma_{t+\tau}\right\}-E\left\{\gamma_{t}\right\} E\left\{\gamma_{t+\tau}\right\}, \\
& =E\left\{\theta_{t+\tau} x_{t} \theta_{t+\tau+\tau^{\prime}} x_{t+\tau^{\prime}}\right\} .
\end{aligned}
$$

From an application of the Isserlis formula $[14,16]$, the following relation results:

$$
\begin{aligned}
r_{t, t+\tau^{\prime}}^{(\gamma)} & =E\left\{\theta_{t+\tau} x_{t} \theta_{t+\tau+\tau^{\prime}} x_{t+\tau^{\prime}}\right\} \\
& =E\left\{\theta_{t+\tau} \theta_{t+\tau+\tau^{\prime}}\right\} E\left\{x_{t} x_{t+\tau^{\prime}}\right\} \\
& =r_{\tau^{\prime}}^{(\theta)} r_{\tau^{\prime}}^{(x)} \\
& =r_{\tau^{\prime}}^{(\gamma)}
\end{aligned}
$$

(11) which is independent of $t$. 


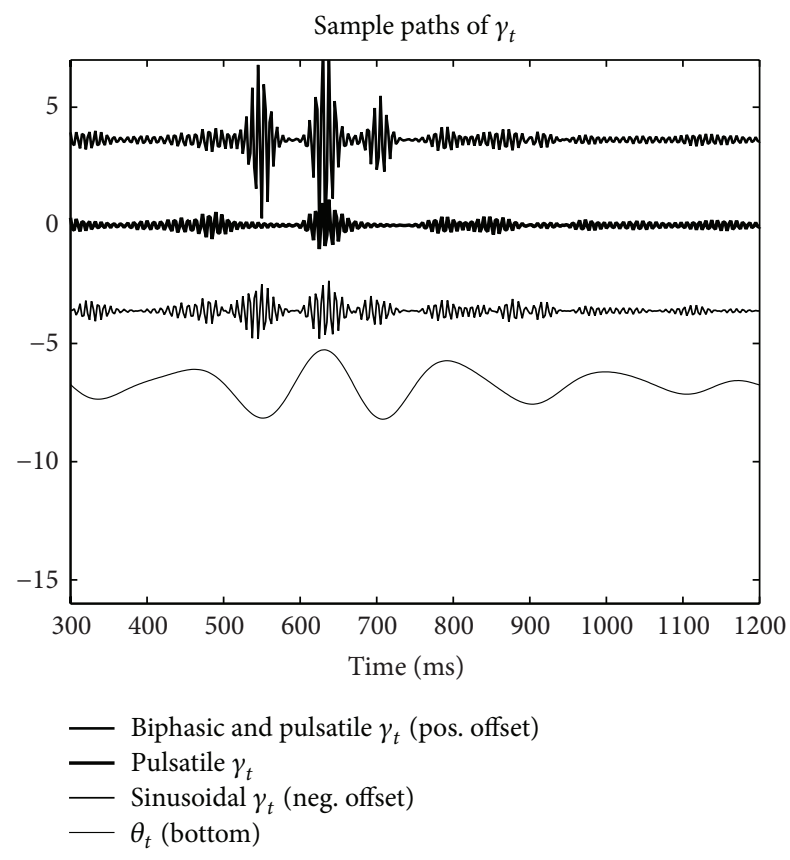

Figure 3: Sample paths of $\gamma$ (scaled). Sinusoidal, pulsatile, and biphasic cross frequency coupling is exhibited. Each $\gamma$ pulse is about 25 milliseconds in duration, consistent with a bandwidth of $40 \mathrm{~Hz}$. These time-series approximate high-pass filtered neural recordings exhibiting CFC.

2.2.4. The Spectrum, $S_{\gamma}$, of $\gamma$. The discrete-time Fourier transform of $r^{(\gamma)}$ is equal to $S_{\gamma}$. From an application of the discrete-time convolution theorem, the following expression relating $S_{\theta}$ and $S_{x}$ to $S_{\gamma}$ is obtained:

$$
S_{\gamma}(f)=\int_{-f_{N}}^{f_{N}} S_{\theta}\left(f^{\prime}\right) S_{x}\left(f-f^{\prime}\right) d f^{\prime} .
$$

2.2.5. Cross Frequency Coupling and the Frequency of $\theta$. To explore the effect of $\theta$ center frequency upon cross frequency coupling, in simulation, two center frequencies are used. These frequencies are $6 \mathrm{~Hz}$ (Figure 4) and $15 \mathrm{~Hz}$ (Figure 5). The effect of making this change can be seen by comparing the spectra plotted in Figure 4 with those plotted in Figure 5.

2.2.6. The Effect of $\gamma$ Bandwidth on Pulse Duration. Since $S_{\gamma}$ is approximately nonzero only on a $40 \mathrm{~Hz}$ interval, the absolute-autocovariance, $\left|r_{t}^{(\gamma)}\right|$, is approximately nonzero for lags of $1 / 40$ seconds. Thus, pulses will tend to have about a 25-millisecond duration.

2.2.7. Simulated Time-Series: Sample Path Generation. To obtain a length $n$ sample-path, that is, a truncated realization, of any one of the random processes $\theta, x$, or $\eta$ described in this work, a draw from $n$ IID standard Gaussian random variables is made (mean-zero, unit variance). These Gaussian random variables can be collected into an $n$-dimensional vector $\mathbf{v}$.

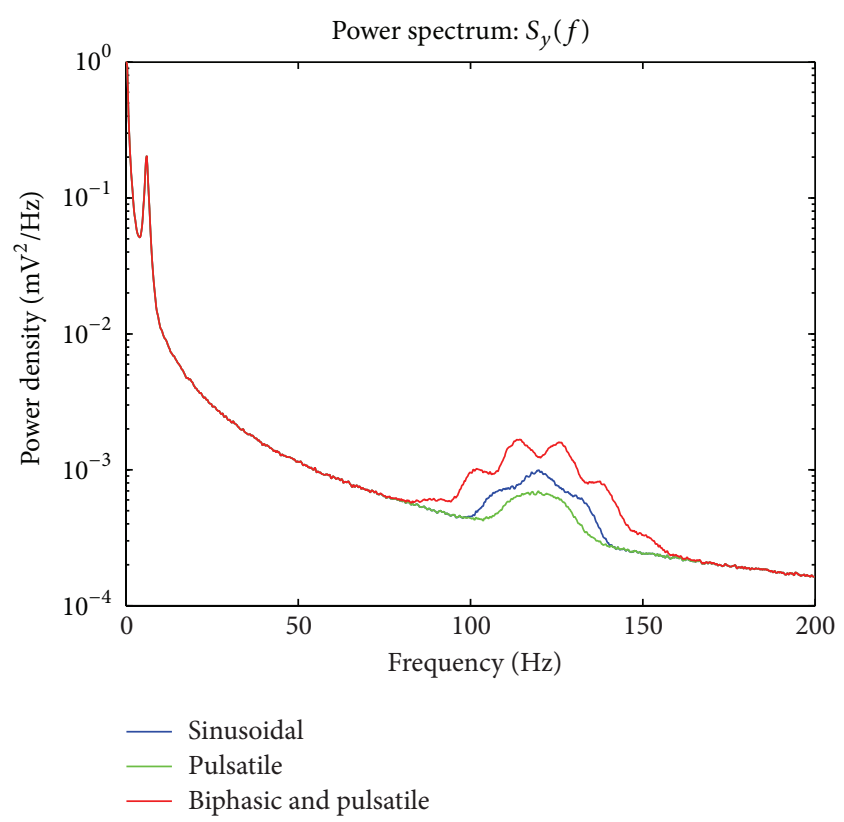

Figure 4: Spectra $S_{y}$ of the stochastic model $y$. The sinusoidal, pulsatile, and biphasic $\gamma$ 's each contribute to the spectrum $S_{y}$ in a different way. The spectrum associated with $\gamma$ computed with the pulsatile function of $\theta$ is similar to the spectrum estimated from actual neural recordings (see [5]). The spectra associated with sinuosoidal, pulsatile, and biphasic $\gamma$ exhibit side-lobes consistent with the convolution appearing in (13), (21).

The difference between $\mathbf{v}$ and the desired random vector $\mathbf{y}$, with covariance matrix $\mathbf{R}$, is

$$
\mathbf{y}=\mathbf{R}^{1 / 2} \mathbf{v}
$$

since

$$
\begin{aligned}
\operatorname{cov}(\mathbf{y}) & =E\left\{\mathbf{y y}^{T}\right\}-E\{\mathbf{y}\} E\left\{\mathbf{y}^{T}\right\}, \\
& =E\left\{\mathbf{y} \mathbf{y}^{T}\right\}, \\
& =\mathbf{R}^{1 / 2} E\left\{\mathbf{v v}^{T}\right\} \mathbf{R}^{1 / 2}, \\
& =\mathbf{R} .
\end{aligned}
$$

Thus, if $\mathbf{R}$ is known, then a realization of $\mathbf{y}$ can be computed by multiplying a realization of $\mathbf{v}$ by $\mathbf{R}^{1 / 2}$. It remains to specify $\mathbf{R}$. For a weak-sense stationary process, the covariance between random variables depends only upon their separation in time-index. Thus, $\mathbf{R}$ is Toeplitz with constant diagonals and is completely specified by the autocovariance function. For example, for the process $y$,

$$
\left(\mathbf{R}^{(y)}\right)_{t, t+\tau}=r_{\tau}^{(y)} .
$$

This method of computing simulated realizations is used for all processes in this work except for $\gamma$, and hence, for $y$. In the former case, a realization of $\gamma$ is computed from realizations of $\theta$ and $x$. Similarly, a realization of $y$ is computed by summing realizations of $\theta, \gamma$, and $\eta$. 


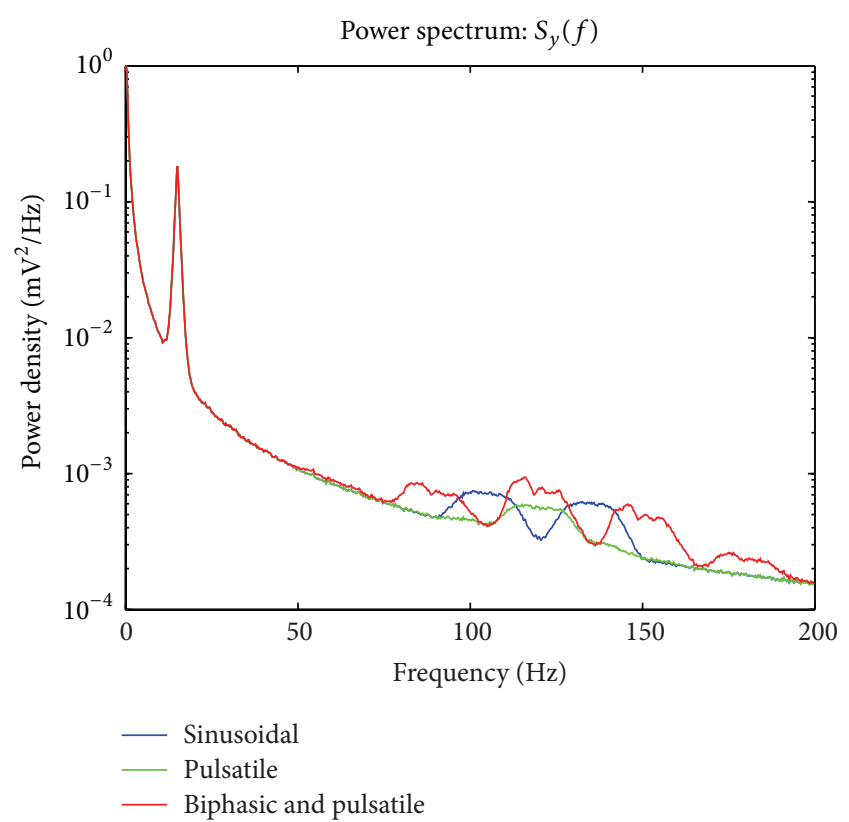

FIGURE 5: Spectra $S_{y}$ of the stochastic model $y$ with $\theta$ centered upon $15 \mathrm{~Hz}$. The sinusoidal, pulsatile, and biphasic $\gamma$ 's each contribute to the spectrum $S_{y}$ in a different way.

2.3. Model Assessment. The appropriateness of $y$ as a model of actual recordings exhibiting CFC is assessed, somewhat crudely, by comparing visually the sample paths of $\gamma$ generated with the three different $f$ functions relating $\theta$ and $x$ to $\gamma$ and by comparing the spectrum of $y$ and $\gamma$ to that exhibited [5] in actual neural recordings.

\section{Results}

3.1. Simulated $\gamma$ Time-Series Exhibiting CFC. Figure 3 depicts sample paths of $\gamma$ constructed, using (5), (6), and (7). Here (5) corresponds to the label "Sinusoidal $\gamma_{t}$ ", (6) corresponds to the label "Pulsatile $\gamma_{t}$," and (7) corresponds to the label "Biphasic $\gamma_{t}$ " These time-series approximate high-pass filtered neural recordings exhibiting CFC.

3.2. The Autocovariance Sequence $r_{y}$. Having established that $y$ is mean-zero, the autocovariance of $y$ is equal to

$$
\begin{aligned}
r_{t, t+\tau}^{(y)} & =E\left\{y_{t} y_{t+\tau}\right\}, \\
& =E\left\{\left[\theta_{t}+\gamma_{t}+\eta_{t}\right] \times\left[\theta_{t+\tau}+\gamma_{t+\tau}+\eta_{t+\tau}\right]\right\} .
\end{aligned}
$$

Due to independence and the fact that $E\left\{\eta_{t}\right\}$ is equal to zero, the following is considered:

$$
\begin{aligned}
r_{t, t+\tau}^{(y)} & =E\left\{\left[\theta_{t}+\gamma_{t}\right]\left[\theta_{t+\tau}+\gamma_{t+\tau}\right]\right\}+r_{\tau}^{(\eta)}, \\
& =r_{\tau}^{(\theta)}+r_{\tau}^{(\gamma)}+E\left\{\theta_{t} \gamma_{t+\tau}\right\}+E\left\{\gamma_{t} \theta_{t+\tau}\right\}+r_{\tau}^{(\eta)},
\end{aligned}
$$

but

$$
\begin{aligned}
E\left\{\theta_{t} \gamma_{t+\tau}\right\} & =E\left\{\theta_{t} \theta_{t+\tau} x_{t+\tau}\right\}, \\
& =E_{\theta_{t}, \theta_{t+\tau}}\left\{E\left\{x_{t+\tau} \mid \theta_{t}, \theta_{t+\tau}\right\}\right\}, \\
& =E_{\theta_{t}, \theta_{t+\tau}}\left\{E\left\{x_{t+\tau}\right\}\right\}, \quad \text { (by independence) } \\
& =0 .
\end{aligned}
$$

Similarly, $E\left\{\gamma_{t} \theta_{t+\tau}\right\}$ is also zero. Then

$$
r_{\tau}^{(y)}=r_{\tau}^{(\theta)}+r_{\tau}^{(\gamma)}+r_{\tau}^{(\eta)}
$$

3.3. $y$ Is Weak-Sense Stationary (WSS). The three components $\theta, \gamma$, and $\eta$ are each mean-zero, and hence $y$ is also mean-zero. Since the autocovariance $r^{(y)}$ is completely specified in (20) in terms of $r^{(\theta)}, r^{(\gamma)}$, and $r^{(\eta)}$, each of which does not depend upon absolute time, $r^{(y)}$ also does not depend upon absolute time. Since the first two moments of the process $y$ are independent of absolute time, $y$ is WSS.

3.4. The Spectrum $S_{y}$ of $y$. Applying (3) to (20) results in the following relation:

$$
\begin{aligned}
S_{y}(f) & =S_{\theta}(f)+S_{\gamma}(f)+S_{\eta}(f), \\
& =S_{\theta}(f)+S_{\eta}(f)+\int_{-1 / 2}^{1 / 2} S_{\theta}\left(f^{\prime}\right) S_{x}\left(f-f^{\prime}\right) d f^{\prime} .
\end{aligned}
$$

3.5. Time-Series Models of Cross Frequency Coupling (CFC). Using the three $\theta, x$ coupling functions (sinusoidal, pulsatile, and biphasic pulsatile) used to specify $\gamma$ and the simulation method discussed in Section 2.2.7, 5000 realizations of $y$ are simulated. From each of these realizations, an estimate of $S_{y}$ is computed. These estimates are averaged to obtain the three curves depicted in Figure 4. The convolution between $f(\theta)$ and $x$ produces prominent side-lobes in the spectra of $y$ for the sinusoidal and biphasic cases. For the pulsatile case the spectrum of $y$ appears similar to those exhibited by actual neural recordings [5].

3.6. $S_{y}$ for a Frequency-Shifted $\theta$. The simulation presented in Section 3.5 is repeated for a modified $\theta$. Specifically, the frequency about which the $\theta$ component is centered is shifted from $6 \mathrm{~Hz}$ to $15 \mathrm{~Hz}$. The resulting spectra associated with the sinusoidal, pulsatile, and biphasic $\theta-\gamma$ coupling are depicted in Figure 5. Sample paths of the associated $\gamma$ are shown in Figure 6.

\section{Discussion}

The stochastic models considered in this work are weaksense stationary. Phenomena without the WSS property, such as stimulus transients and time varying changes in autocovariance, are not captured by these models. While the topic is left for future study, one expects a convolution similar 


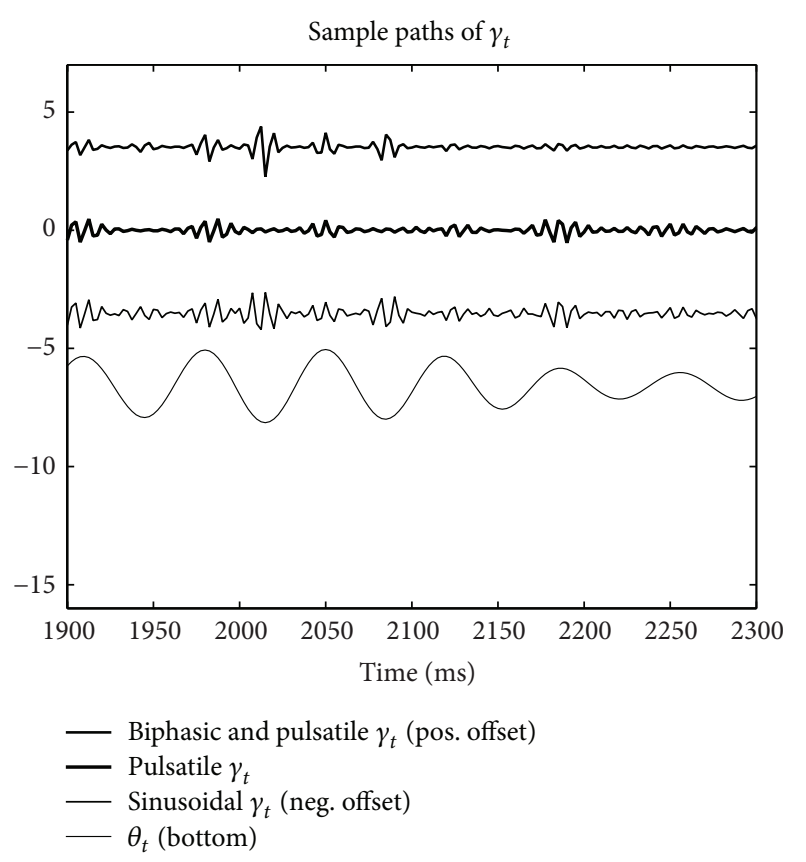

Figure 6: Sample paths of $\gamma$ (scaled). In this case $\gamma$ is coupled to a $15 \mathrm{~Hz} \theta$. For comparison with a $\theta$ centered about $6 \mathrm{~Hz}$ see Figure 3. Sinusoidal, pulsatile, and biphasic cross frequency coupling is exhibited. Each $\gamma$ pulse is about 25 milliseconds in duration, consistent with a bandwidth of $40 \mathrm{~Hz}$. These time-series approximate high-pass filtered neural recordings exhibiting CFC.

to that producing $\gamma$ to appear in the nonstationary analogs of the time-series models considered in this work.

In [17] the probability density function for a phaseamplitude coupling estimator is provided under the assumption that phase-amplitude CFC is absent. The parametric regression based phase-amplitude coupling estimators [18, 19] are based upon conditional expectations which may not completely reflect the statistical properties of recordings that exhibit phase-amplitude coupling. The time-series models explicitly proposed in this work account for realistic temporal dependency and exhibit phase-amplitude CFC. As in [17], sampling properties of proposed estimators can be assessed based on a time-series model.

Owing to the convolution integral present in (13) and (21), the time-series introduced in this work exhibit, in general, multiple peaks about the $120 \mathrm{~Hz}$ center frequency of $\gamma$. This effect is minimized for CFC developed through the product of the latent process $x$ with the pulsatile function $f(\theta)$ specified in (6). Of the considered models, it is this latter model which most closely matches observed CFC phenomena. The pulsatile function can be loosely thought of as imposing a window over each cycle of the slow oscillation during which the latent and relatively high-frequency process $x$ is amplified. In the neural context, one might infer the existence of a similar window defining intervals of time when neural activity is more easily generated, consistent with the notion that oscillatory activity represents cyclic changes in excitability [3].
The excitability described in [3] is considered to be a function of the relative levels of synaptic inhibition and excitation. Pulsatile functions that more closely match the shape and time-course of synaptic currents may more closely match the observed features of phase-amplitude CFC in neural data. By comparing the simulated data resulting from the shape and time course of a simulated pulsatile function to actual neural data, insight may be gleaned regarding the nature of synaptic currents underlying phase-amplitude CFC. This modeling process, beginning with a stochastic time-series model of observed phenomena, may also provide insight into the other types of CFC, namely, amplitudeamplitude and phase-phase cross frequency coupling.

The process of time-series model simulation and the comparison of simulation results to results obtained from neural data can be facilitated with the use of statistical methods. The quantities in the proposed time-series models can be estimated, confidence intervals can be provided, model selection can be performed, and across-condition comparisons and hypothesis tests can be developed. Work refining these models and developing associated statistical methodology is underway.

The time-series models introduced in this work are, to the best of the authors' knowledge, the first reported time-series models capable of generating phase-amplitude cross frequency coupling. They may provide a basis for a more accurate and statistically principled assessment of cross frequency coupling and underlying synaptic activity that accounts for the temporal dependencies present in actual recordings.

Finally, the method of simulating sample-paths of weaksense stationary processes introduced in Section 2.2.7 seems to be novel to the neuroscience community. Its use may facilitate increased accuracy when numerically investigating the behavior of statistics computed from neural recordings.

\section{Conclusion}

Full appreciation of the effect of different time-domain manifestations of cross frequency coupling may provide insight into neural processes. In particular, prominent spectral features are produced by somewhat mild changes to the nature of a time-series model exhibiting phase-amplitude cross frequency coupling. These changes may provide the basis for refined statistical methodology capable of shedding light into the nature of the inhibitory and excitatory synaptic currents supporting phase-amplitude cross frequency coupling.

\section{Appendix}

\section{DTFT of a Periodic Sequence}

Let $g$ be a sequence with discrete-time Fourier transform (DTFT) G. Then

$$
g_{t}=\int_{-f_{N}}^{f_{N}} G\left(f^{\prime}\right) e^{i 2 \pi f^{\prime} t \Delta}
$$


Further, let $G(f)$ be nonzero only for $f=n / \tau \Delta, n=-4$, $-3, \ldots, 3,4$. Then

$$
\begin{aligned}
g_{t} & =\int_{-f_{N}}^{f_{N}} G\left(f^{\prime}\right) e^{i 2 \pi f^{\prime} t \Delta}, \\
& =G(0)+\sum_{\substack{n=-4 \\
n \neq 0}}^{4} G\left(\frac{n}{\tau \Delta}\right) e^{i 2 n \pi(t / \tau)} .
\end{aligned}
$$

Because for each $n, e^{i 2 \pi n(t / \tau)}$ is periodic with period $\tau, g$ is also periodic with period $\tau$.

\section{Conflict of Interests}

The authors declare that they have no competing interests.

\section{Acknowledgment}

The authors are supported by NSF Grant DMS-1042134.

\section{References}

[1] C. T. Dickson, G. Biella, and M. De Curtis, "Evidence for spatial modules mediated by temporal synchronization of carbacholinduced gamma rhythm in medial entorhinal cortex," The Journal of Neuroscience, vol. 20, no. 20, pp. 7846-7854, 2000.

[2] A. von Stein and J. Sarnthein, "Different frequencies for different scales of cortical integration: from local gamma to long range alpha/theta synchronization," International Journal of Psychophysiology, vol. 38, no. 3, pp. 301-313, 2000.

[3] P. Lakatos, A. S. Shah, K. H. Knuth, I. Ulbert, G. Karmos, and C. E. Schroeder, "An oscillatory hierarchy controlling neuronal excitability and stimulus processing in the auditory cortex," Journal of Neurophysiology, vol. 94, no. 3, pp. 1904-1911, 2005.

[4] R. T. Canolty, E. Edwards, S. S. Dalal et al., "High gamma power is phase-locked to theta oscillations in human neocortex," Science, vol. 313, no. 5793, pp. 1626-1628, 2006.

[5] C. Scheffzük, V. I. Kukushka, A. L. Vyssotski, A. Draguhn, A. B. L. Tort, and J. Brankačk, "Selective coupling between theta phase and neocortical fast gamma oscillations during REMsleep in mice," PLoS ONE, vol. 6, no. 12, Article ID e28489, 2011.

[6] A. B. L. Tort, R. W. Komorowski, J. R. Manns, N. J. Kopell, and H. Eichenbaum, "Theta-gamma coupling increases during the learning of item-context associations," Proceedings of the National Academy of Sciences of the United States of America, vol. 106, no. 49, pp. 20942-20947, 2009.

[7] J. J. Chrobak and G. Buzsáki, "Gamma oscillations in the entorhinal cortex of the freely behaving rat," The Journal of Neuroscience, vol. 18, no. 1, pp. 388-398, 1998.

[8] A. B. L. Tort, M. A. Kramer, C. Thorn et al., "Dynamic crossfrequency couplings of local field potential oscillations in rat striatum and hippocampus during performance of a T-maze task," Proceedings of the National Academy of Sciences of the United States of America, vol. 105, no. 51, pp. 20517-20522, 2008.

[9] B. Voytek, R. T. Canolty, A. Shestyuk, N. E. Crone, J. Parvizi, and R. T. Knight, "Shifts in gamma phase-amplitude coupling frequency from theta to alpha over posterior cortex during visual tasks," Frontiers in Human Neuroscience, vol. 4, article 191, 2010.
[10] J. Brankačk, C. Scheffzük, V. I. Kukushka, A. L. Vyssotski, A. B. L. Tort, and A. Draguhn, "Distinct features of fast oscillations in phasic and tonic rapid eye movement sleep," Journal of Sleep Research, vol. 21, no. 6, pp. 630-633, 2012.

[11] P. Lakatos, G. Karmos, A. D. Mehta, I. Ulbert, and C. E. Schroeder, "Entrainment of neuronal oscillations as a mechanism of attentional selection," Science, vol. 320, no. 5872, pp. 110113, 2008.

[12] J. E. Lisman and O. Jensen, “The $\theta-\gamma$ neural code," Neuron, vol. 77, no. 6, pp. 1002-1016, 2013.

[13] M. B. Priestley, Spectral Analysis and Time Series, Elsevier Academic Press, 1981.

[14] D. B. Percival and A. T. Walden, Spectral Analysis for Physical Applications, Cambridge University Press, Cambridge, UK, 1993.

[15] D. R. Brillinger, Time Series: Data Analysis and Theory, vol. 36 of Classics in Applied Mathematics, Society for Industrial and Applied Mathematics, Philadelphia, Pa, USA, 2001.

[16] L. Isserlis, "On a formula for the product-moment coefficient of any order of a normal frequency distribution in any number of variables," Biometrika, vol. 12, no. 1-2, pp. 134-139, 1918.

[17] T. E. Özkurt, "Statistically reliable and fast direct estimation of phase-amplitude cross-frequency coupling," IEEE Transactions on Biomedical Engineering, vol. 59, no. 7, pp. 1943-1950, 2012.

[18] W. D. Penny, E. Duzel, K. J. Miller, and J. G. Ojemann, “Testing for nested oscillation," Journal of Neuroscience Methods, vol. 174, no. 1, pp. 50-61, 2008.

[19] M. A. Kramer and U. T. Eden, "Assessment of cross-frequency coupling with confidence using generalized linear models," Journal of Neuroscience Methods, vol. 220, no. 1, pp. 64-74, 2013. 

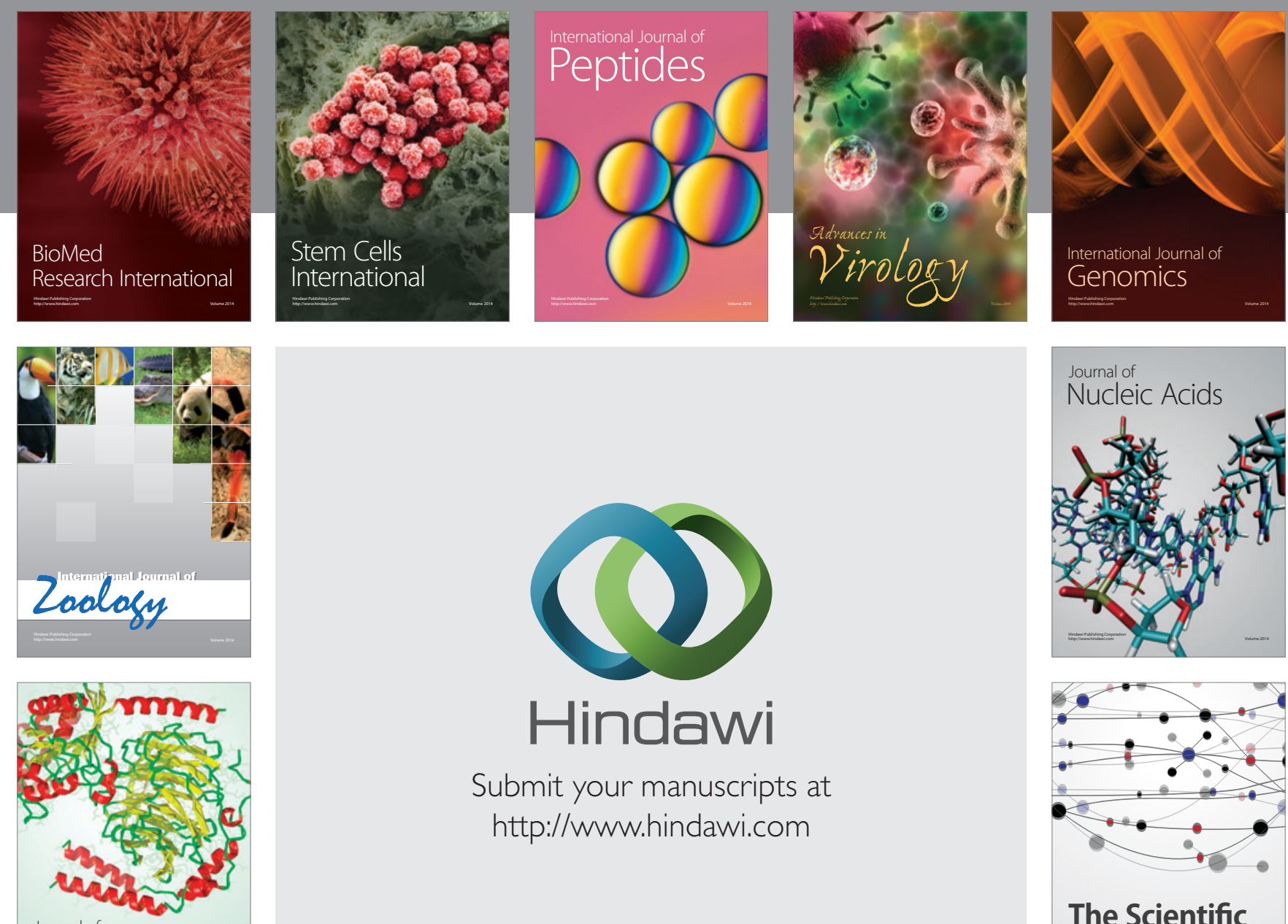

Submit your manuscripts at

http://www.hindawi.com

Journal of
Signal Transduction
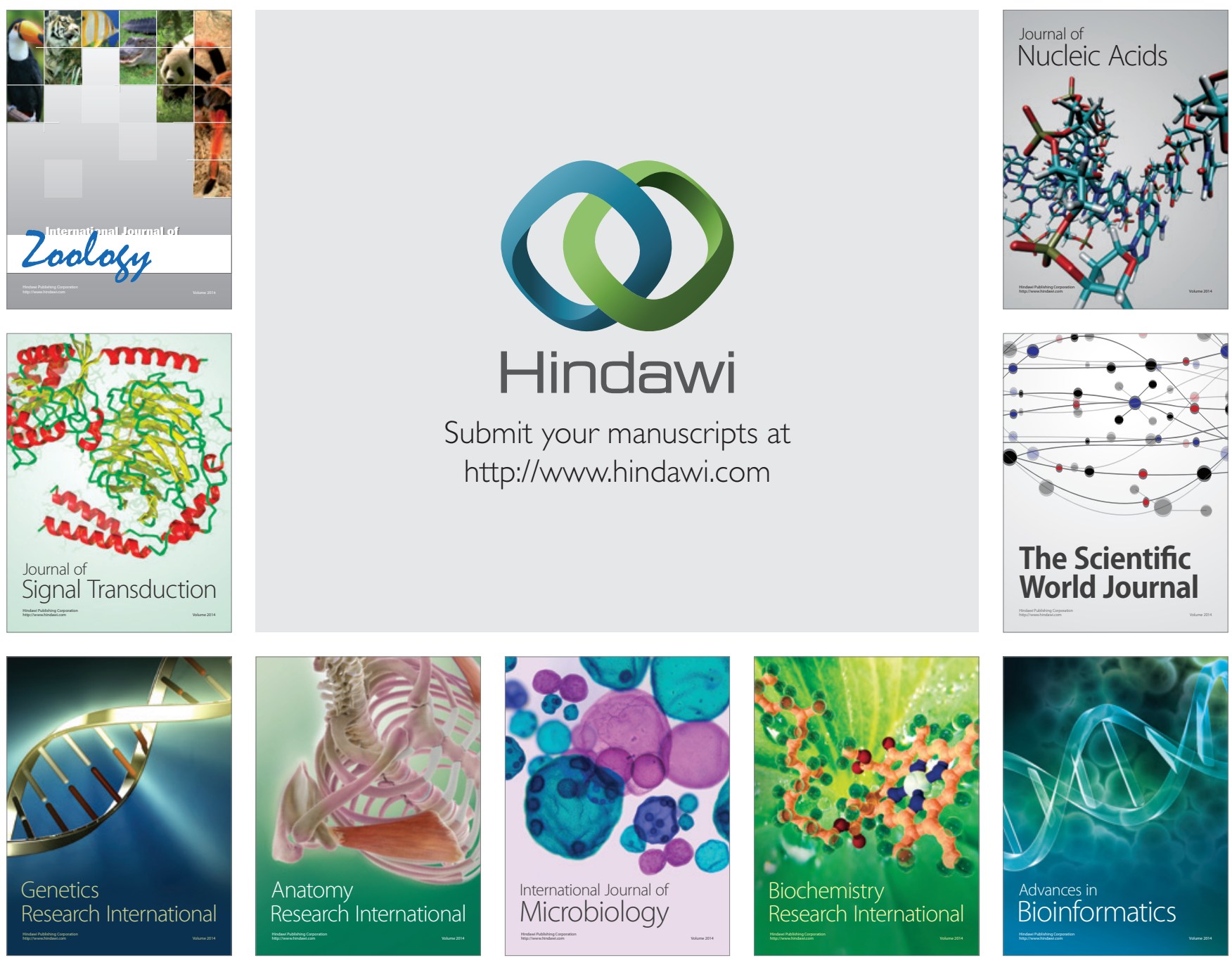

The Scientific World Journal
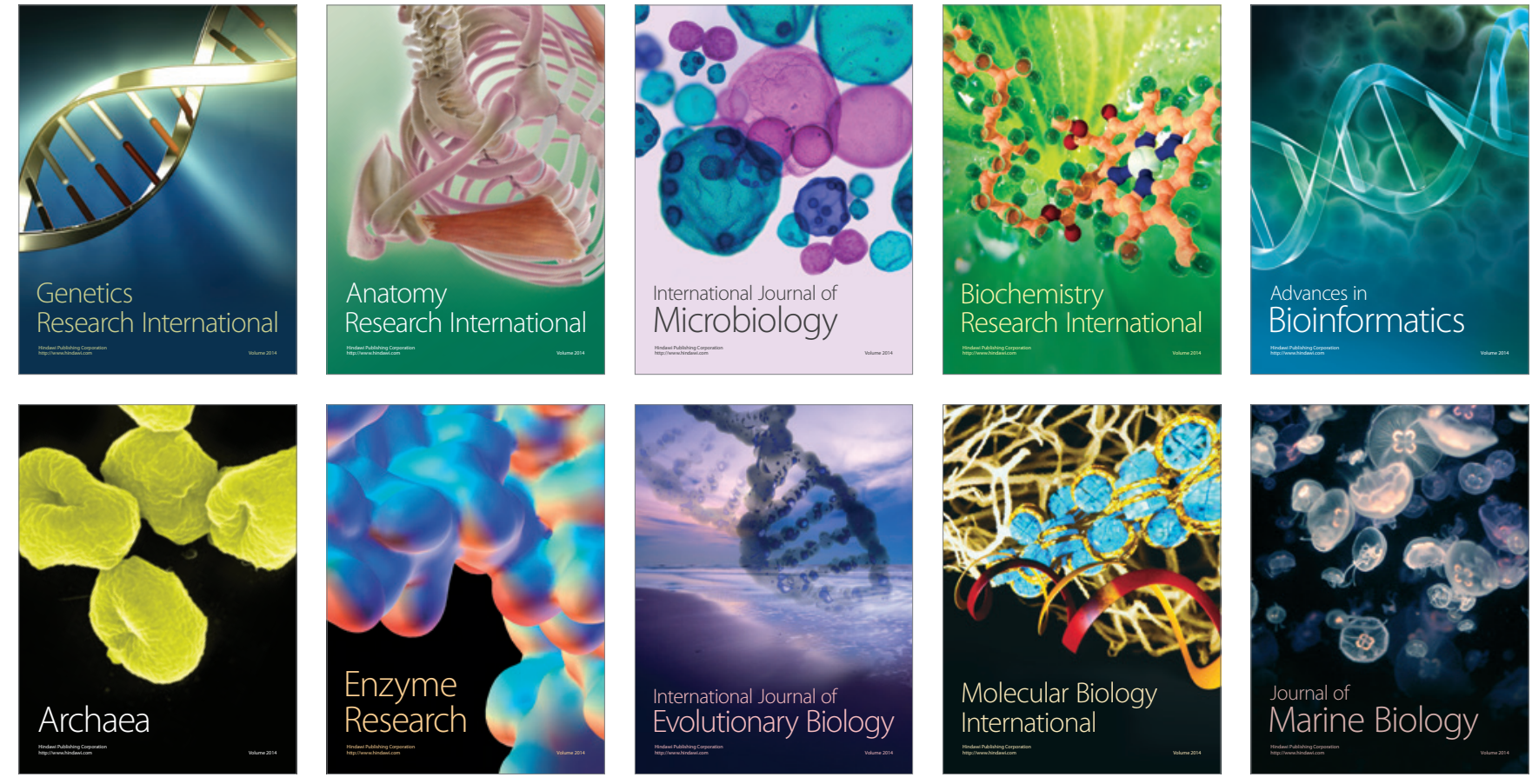